\title{
A Constructive Model of Uniform Continuity
}

\author{
Chuangjie Xu and Martín Escardó \\ University of Birmingham, UK
}

\begin{abstract}
We construct a continuous model of Gödel's system T and its logic $\mathrm{HA}^{\omega}$ in which all functions from the Cantor space $\mathbf{2}^{\mathbb{N}}$ to the natural numbers are uniformly continuous. Our development is constructive, and has been carried out in intensional type theory in Agda notation, so that, in particular, we can compute moduli of uniform continuity of T-definable functions $\mathbf{2}^{\mathbb{N}} \rightarrow \mathbb{N}$. Moreover, the model has a continuous Fan functional of type $\left(\mathbf{2}^{\mathbb{N}} \rightarrow \mathbb{N}\right) \rightarrow \mathbb{N}$ that calculates moduli of uniform continuity. We work with sheaves, and with a full subcategory of concrete sheaves that can be presented as sets with structure, which can be regarded as spaces, and whose natural transformations can be regarded as continuous maps.
\end{abstract}

Keywords. Constructive mathematics, topological models, uniform continuity, Fan functional, intuitionistic type theory, topos theory, sheaves, HA $^{\omega}$, Gödel's system T.

\section{Introduction}

Gödel's system T has a well-known topological models in which all integer-valued functions on the Cantor space are uniformly continuous:

$$
\forall f: \mathbf{2}^{\mathbb{N}} \rightarrow \mathbb{N} . \exists n \in \mathbb{N} . \forall \alpha, \beta \in \mathbf{2}^{\mathbb{N}} . \alpha={ }_{n} \beta \Longrightarrow f(\alpha)=f(\beta),
$$

where $\alpha={ }_{n} \beta$ means $\forall i<n$. $\alpha_{i}=\beta_{i}$. These models include Kleene-Kreisel functionals [19], compactly generated spaces [19], limit spaces [20], equilogical spaces [3], sequential spaces [12], and QCB spaces [12]. However, even though these models are introduced for the purposes of computability theory, they are developed within a classical meta-theory.

The purpose of this paper is to develop such a topological model in a weak constructive meta-theory, without explicit reference to computability, but with computability in mind. In fact, we conjecture that our model is classically equivalent to the model of Kleene-Kreisel spaces. Our continuous model of system T consists of certain C-spaces, which can be seen as sheaves (see below), and is developed in Section 2. Like the above models, this model has a Fan functional of type $\left(2^{\mathbb{N}} \rightarrow \mathbb{N}\right) \rightarrow \mathbb{N}$ that continuously calculates moduli of uniform continuity (Section 3). Importantly, we do not rely on the Fan Theorem [5] or any such principle to construct the Fan functional. In particular, we recover the wellknown fact that the T-definable functions $\mathbf{2}^{\mathbb{N}} \rightarrow \mathbb{N}$ are uniformly continuous [5] by defining a logical relation between the set-theoretical and continuous models (Section 4). To model the logic HA ${ }^{\omega}$ extended with the above uniform continuity 
axiom, we realize the logical operations by continuous functions in our model (Section 5). We also discuss how our results can be extended to give a continuous model of dependent types (Section 7).

As mentioned above, we develop this in a weak constructive meta-language, which does not incorporate constructively contentious axioms such as continuity principles, Fan Theorem, Bar Induction, or Church's Thesis [5]. In this presentation we deliberately leave the details of the meta-language unexplained, relying on the readers' ability to recognize constructive arguments. One possible formal constructive meta-language for our development is intensional Martin-Löf type theory (MLTT), and, in fact, we have developed the main results of this paper in Agda [6] (Section 6). Because MLTT has a computational interpretation, our model can be used to compute moduli of uniform continuity of system $\mathrm{T}$ definable functions $\mathbf{2}^{\mathbb{N}} \rightarrow \mathbb{N}$. More importantly, our model can be used to extract computational content from proofs in $\mathrm{HA}^{\omega}$ extended with the above uniform continuity axiom.

Our model is a sheaf topos, with a full subcategory of concrete sheaves [2] that can be presented as sets with structure, which can be regarded as spaces, and whose natural transformations can be regarded as continuous maps. The underlying category of our site is the monoid of uniformly continuous endomaps of the Cantor space, with a natural coverage consisting of families of concatenation maps. The coverage axiom amounts precisely to the uniform continuity of the elements of our monoid. Our development of this topos is fairly standard, but we have taken care of making sure the arguments are presented in a form suitable for a formalization in a predicative type theory, and this is one of the main contributions of this work.

Our work builds upon Johnstone's paper On a topological topos (1979), Fourman's papers Continuous truth and Notions of choice sequence (1982), van der Hoeven and Moerdijk's paper Sheaf models for choice sequences (1984), Bauer and Simpson's unpublished work Continuity begets continuity (2006), and Coquand and Jaber's paper A note on forcing and type theory (2010) and A computational interpretation of forcing in type theory (2012).

Johnstone, Fourman, van der Hoeven and Moerdijk's work with sheaf toposes over different sites. Johnstone's site is the monoid of continuous endo-functions of the one-point compactification of the discrete natural numbers with the canonical Grothendieck topology. A full subcategory of Johnstone's topological topos is that of sequential topological spaces, which is cartesian closed. A bigger full subcategory, which is locally cartesian closed, is that of Kuratowski limit spaces. The concrete sheaves, or spaces, in our model correspond to the Kuratowski limit spaces in Johnstone's construction, and are also related to Spanier's quasitopological spaces [22], as we discuss in the body of the paper. Bauer and Simpson's work can be seen as taking place in the topological topos.

Fourman works with a site whose underlying category is the semilattice of finite sequences of natural numbers under the prefix order, and van der Hoeven and Moerdijk work with a site whose underlying category is the monoid of continuous endomaps of the Baire space, and they relate their work to Fourman's. 
Coquand and Jaber's forcing model instead uses the semilattice of finite binary sequences under the prefix order as the underlying category of the site, modelling the idea of a generic infinite binary sequence. They iterate their construction in order to be able to model the Fan functional, and our model can be regarded as accomplishing this iteration directly (personal communication with Coquand).

\section{Sheaves and Spaces}

\subsection{Sheaves and Natural Transformations}

Recall that a presheaf on a small category $\mathbf{C}$ is a functor $\mathbf{C}^{\mathrm{op}} \rightarrow$ Set. When $\mathbf{C}$ is a one-object category, i.e. a monoid, this can be formulated in terms of monoid actions $[18, \S \mathrm{I} .1]$. A presheaf on a monoid $(\mathbf{C}, \circ, \mathrm{id})$ amounts to a set $P$ with an action

$$
((p, t) \mapsto p \cdot t): P \times \mathbf{C} \rightarrow P
$$

such that for all $p \in P$ and $t, u \in \mathbf{C}$

$$
p \cdot \mathrm{id}=p, \quad p \cdot(t \circ u)=(p \cdot t) \cdot u .
$$

A natural transformation of presheaves $(P, \cdot)$ and $(Q, \cdot)$ amounts to a function $\phi: P \rightarrow Q$ that preserves the action, i.e.

$$
\phi(p \cdot t)=(\phi p) \cdot t
$$

We work with the monoid $\mathrm{C}$ of uniformly continuous endo-maps on the Cantor space $\mathbf{2}^{\mathbb{N}}$, that is, the functions $t: \mathbf{2}^{\mathbb{N}} \rightarrow \mathbf{2}^{\mathbb{N}}$ such that

$$
\forall m \in \mathbb{N} . \exists n \in \mathbb{N} . \forall \alpha, \beta \in \mathbf{2}^{\mathbb{N}} . \alpha={ }_{n} \beta \Longrightarrow t(\alpha)={ }_{m} t(\beta) .
$$

Notice that any continuous function $\mathbf{2}^{\mathbb{N}} \rightarrow \mathbf{2}^{\mathbb{N}}$ is uniformly continuous, assuming classical logic or the Fan Theorem. Because we do not assume such principles, we need to explicitly require uniform continuity in the definition of the monoid $\mathrm{C}$.

Our site is the monoid $\mathrm{C}$ equipped with the countable coverage $\mathcal{J}$ consisting of the finite covering families

$$
\left\langle\operatorname{cons}_{s}\right\rangle_{s \in 2^{n}}
$$

for all natural numbers $n$, where $\mathbf{2}^{n}$ is the set of binary sequences of length $n$ and cons $_{s}: \mathbf{2}^{\mathbb{N}} \rightarrow \mathbf{2}^{\mathbb{N}}$ is the concatenation map:

$$
\operatorname{cons}_{s}(\alpha)=s \alpha .
$$

It is easy to verify that, for any $n \in \mathbb{N}$ and for any $s \in \mathbf{2}^{n}$, the concatenation map cons $s$ is uniformly continuous and so cons $_{s} \in \mathrm{C}$.

The coverage axiom specialized to our situation amounts to saying that for all $t \in \mathrm{C}$,

$$
\forall m \in \mathbb{N} . \exists n \in \mathbb{N} . \forall s \in \mathbf{2}^{n} . \exists t^{\prime} \in \mathrm{C} . \exists s^{\prime} \in \mathbf{2}^{m} . t \circ \operatorname{cons}_{s}=\operatorname{cons}_{s^{\prime}} \circ t^{\prime} .
$$

It is routine to show that: 
Lemma 1. A map $t: \mathbf{2}^{\mathbb{N}} \rightarrow \mathbf{2}^{\mathbb{N}}$ satisfies ( $\dagger$ ) iff it is uniformly continuous.

Thus, not only does the coverage axiom hold, but also it amounts to the fact that the elements of the monoid $\mathrm{C}$ are the uniformly continuous functions. Notice that every covering family is jointly surjective. Because the maps in each covering family have disjoint images, we do not need to consider the compatibility condition in the definition of sheaf:

Lemma 2. A presheaf $(P, \cdot)$ is a sheaf iff for every $n \in \mathbb{N}$ and every family $\left\langle p_{s} \in P\right\rangle_{s \in \mathbf{2}^{n}}$, there is a unique amalgamation $p \in P$ such that, for all $s \in \mathbf{2}^{n}$,

$$
p \cdot \operatorname{cons}_{s}=p_{s} .
$$

Notice also that, by induction, it is enough to consider the case $n=1$ :

Lemma 3. A presheaf $(P, \cdot)$ is a sheaf iff for any two $p_{0}, p_{1} \in P$, there is a unique $p \in P$ such that

$$
p \cdot \operatorname{cons}_{0}=p_{0} \quad \text { and } \quad p \cdot \text { cons }_{1}=p_{1} .
$$

This construction gives a full subcategory $\operatorname{Shv}(\mathrm{C}, \mathcal{J})$ of the category of presheaves, consisting of the sheaves over the site $(\mathrm{C}, \mathcal{J})$.

\subsection{Spaces and Continuous Maps}

An important example of a sheaf is the monoid $\mathrm{C}$ itself with function composition as the action. Given $t_{0}, t_{1} \in \mathrm{C}$, the amalgamation $t: \mathbf{2}^{\mathbb{N}} \rightarrow \mathbf{2}^{\mathbb{N}}$ is simply

$$
t(\alpha)=t_{\alpha_{0}}\left(\lambda n . \alpha_{n+1}\right) .
$$

We say a presheaf is concrete if its action is function composition. Then all the elements in a concrete presheaf $(P, \circ)$ must be maps from the Cantor space to some set $X$. Concrete sheaves admit a more concrete description as the set $X$ with the additional structure given by the maps in $P$. We denote the full subcategory of concrete sheaves by $\operatorname{CShv}(\mathrm{C}, \mathcal{J})$.

Concrete sheaves can be regarded as spaces, and their natural transformations as continuous maps. More precisely, they are analogous to Spanier's quasitopological spaces [22], which have the category of topological spaces and continuous maps as a full subcategory. One advantage of quasi-topological spaces over topological spaces, which is the main reason for Spanier's introduction of the notion of quasi-space, is that continuous maps of quasi-spaces form a cartesian closed category. This category serves as a model of system $T$ and $\mathrm{HA}^{\omega}$ that validates the uniform continuity principle, assuming classical logic in the metalanguage. Our concrete sheaves can be seen as analogues of quasi-topological spaces, admitting a constructive treatment.

A quasi-topology on a set $X$ assigns to each compact Hausdorff space $K$ a set $P(K, X)$ of functions $K \rightarrow X$ such that:

(1) All constant maps are in $P(K, X)$. 
(2) If $t: K^{\prime} \rightarrow K$ is continuous and $p \in P(K, X)$, then $p \circ t \in P\left(K^{\prime}, X\right)$.

(3) If $\left\langle t_{i}: K_{i} \rightarrow K\right\rangle_{i \in I}$ is a finite, jointly surjective family and $p: K \rightarrow X$ is a map with $p \circ t_{i} \in P\left(K_{i}, X\right)$ for every $i \in I$, then $p \in P(K, X)$.

A quasi-topological space is a set endowed with a quasi-topology, and a continuous map of quasi-spaces $(X, P)$ and $(Y, Q)$ is a function $f: X \rightarrow Y$ such that $f \circ p \in Q(K, Y)$ whenever $p \in P(K, X)$. For example, every topological space $X$ is a quasi-topological space with the quasi-topology $P$ such that $P(K, X)$ is the set of continuous maps $K \rightarrow X$, and this construction gives the full embedding of topological spaces into quasi-topological spaces.

This definition can be modified by considering just one compact Hausdorff space, the Cantor space, rather than all compact Hausdorff spaces, and by restricting the jointly surjective finite families of continuous maps to the covering families $\left\langle\text { cons }_{s}\right\rangle_{s \in \mathbf{2}^{n}}$ considered in the previous section. We call the resulting objects C-spaces.

Definition 1. A C-space is a set $X$ equipped with a $\mathrm{C}$-topology $P$, i.e. a collection of maps $\mathbf{2}^{\mathbb{N}} \rightarrow X$, called probes, satisfying the following conditions:

(1) All constant maps are in $P$.

(2) (Presheaf condition) If $p \in P$ and $t \in \mathrm{C}$, then $p \circ t \in P$.

(3) (Sheaf condition) For any $n \in \mathbb{N}$ and any family $\left\langle p_{s} \in P\right\rangle_{s \in \mathbf{2}^{n}}$, the unique map $p: \mathbf{2}^{\mathbb{N}} \rightarrow X$ defined by $p(s \alpha)=p_{s}(\alpha)$ is in $P$.

$A$ continuous map of $\mathrm{C}$-spaces $(X, P)$ and $(Y, Q)$ is a map $f: X \rightarrow Y$ with $f \circ p \in Q$ whenever $p \in P$. We write $\mathrm{C}$-Space for the category of $\mathrm{C}$-spaces and continuous maps. The above three conditions are called the probe axioms.

Notice that the sheaf condition is equivalent to

$\left(3^{\prime}\right)$ If $p: \mathbf{2}^{\mathbb{N}} \rightarrow X$ is a map such that there exists $n \in \mathbb{N}$ with $p \circ$ cons $_{s} \in P$ for all $s \in \mathbf{2}^{n}$, then $p \in P$.

The idea is that we "topologize" the set $X$ by choosing a designated set $P$ of maps $2^{\mathbb{N}} \rightarrow X$ that we want, and hence declare, to be continuous. For example, if $X$ already has some form of topology, e.g. a metric, we can take $P$ to be the set of continuous functions $2^{\mathbb{N}} \rightarrow X$ with respect to this topology and the natural topology of the Cantor space. Of course we have to make sure the sheaf condition is satisfied.

As mentioned earlier, C-spaces provide a more concrete description of concrete sheaves in the following sense. Given a $\mathrm{C}$-space $(X, P)$, the $\mathrm{C}$-topology $P$ together with function composition is a concrete sheaf. Conversely, if $(P, \circ)$ is a concrete sheaf, then all maps in $P$ should have the same codomain.

Proposition 1. The two categories $\mathrm{C}-\mathrm{Space}$ and $\operatorname{CShv}(\mathrm{C}, \mathcal{J})$ are naturally equivalent.

By virtue of this equivalence, C-Space can also be viewed as a full subcategory of $\operatorname{Shv}(\mathrm{C}, \mathcal{J})$. Moreover, C-spaces are closed under products and form an exponential ideal. 
To improve the readability, we abbreviate $X$ for the space $(|X|$, Probe $(X))$ where $|X|$ stands for the underlying set and $\operatorname{Probe}(X)$ for the collection of probes, i.e. the C-topology on $|X|$, and we often write $X$ to mean $|X|$ by an abuse of notation.

\subsection{The Cartesian Closed Structure of C-Space}

C-spaces have several convenient categorical properties, the first of which is cartesian closedness.

Theorem 1. The category C-Space of is cartesian closed.

Proof. Any singleton set $\mathbf{1}=\{\star\}$ with the unique map $\mathbf{2}^{\mathbb{N}} \rightarrow \mathbf{1}$ as the only probe is a $\mathrm{C}$-space as well as a terminal object in C-Space.

Given C-spaces $(X, P)$ and $(Y, Q)$, their product is the cartesian product $X \times Y$ equipped with the collection $R$ of probes defined by the condition that $r: \mathbf{2}^{\mathbb{N}} \rightarrow X \times Y$ is in $R$ iff $\pi_{0} \circ r \in P$ and $\pi_{1} \circ r \in Q$, where $\pi_{0}$ and $\pi_{1}$ are the projections. We have to verify that $R$ satisfies the probe axioms and that this has the universal property of a categorical product in C-Space, i.e. continuity of projection functions and its universal property, but this is routine.

Given C-spaces $(X, P)$ and $(Y, Q)$, their exponential is the set $Y^{X}$ of continuous maps $X \rightarrow Y$ equipped with the collection $R$ of probes defined by the condition that $r: \mathbf{2}^{\mathbb{N}} \rightarrow Y^{X}$ is in $R$ iff for any $t \in \mathrm{C}$ and $p \in P$ the map $\lambda \alpha . r(t \alpha)(p \alpha)$ is in $Q$. Again, we have to verify that the probe axioms are satisfied and that this has the universal property of an exponential in C-Space, which involves some subtleties regarding the coverage axiom.

Theorem 2. The category C-Space has finite coproducts.

Proof. The empty set equipped with the empty collection of probes is a C-space and an initial object in C-Space.

Binary coproducts can be constructed as follows: given C-spaces $(X, P)$ and $(Y, Q)$, their coproduct is the disjoint union $X+Y$ equipped with the collection $R$ of probes defined by the condition that $r: \mathbf{2}^{\mathbb{N}} \rightarrow X+Y$ is in $R$ iff there exists $n \in \mathbb{N}$ such that for all $s \in \mathbf{2}^{n}$ either there exists $p \in P$ with $r\left(\operatorname{cons}_{s} \alpha\right)=\operatorname{in}_{0}(p \alpha)$ for all $\alpha \in \mathbf{2}^{\mathbb{N}}$ or there exists $q \in Q$ with $r\left(\operatorname{cons}_{s} \alpha\right)=\operatorname{in}_{1}(q \alpha)$ for all $\alpha \in \mathbf{2}^{\mathbb{N}}$. We have to verify that the probe axioms are satisfied and that this has the required universal property.

\subsection{Discrete C-spaces and Natural Numbers Object}

We say that a C-space $X$ is discrete if for every C-space $Y$, all functions $X \rightarrow Y$ are continuous. A map $p: \mathbf{2}^{\mathbb{N}} \rightarrow X$ into a set $X$ is called locally constant iff

$$
\exists n \in \mathbb{N} . \forall \alpha, \beta \in \mathbf{2}^{\mathbb{N}} . \alpha={ }_{n} \beta \Longrightarrow p(\alpha)=p(\beta) .
$$


Lemma 4. Let $X$ be any set.

(1) The locally constant functions $2^{\mathbb{N}} \rightarrow X$ form a C-topology on $X$.

(2) For any C-topology $P$ on $X$, every locally constant function $2^{\mathbb{N}} \rightarrow X$ is in $P$.

In other words, the locally constant maps $2^{\mathbb{N}} \rightarrow X$ form the smallest C-topology on the set $X$. Moreover:

Lemma 5. A C-space is discrete iff the probes on it are precisely the locally constant functions.

We thus refer to the collection of locally constant maps $\mathbf{2}^{\mathbb{N}} \rightarrow X$ as the discrete C-topology on $X$. In particular, when the set $X$ is $\mathbf{2}$ or $\mathbb{N}$, the locally constant functions amount to the uniformly continuous functions. Hence we have a discrete two-point space $\mathbf{2}$ and a discrete space $\mathbb{N}$ of natural numbers, which play an important role in our model:

Theorem 3. In the category C-Space:

1. The coproduct of two copies of the terminal space $\mathbf{1}$ is the discrete two-point space 2 .

2. The discrete space of natural numbers is the natural numbers object.

Proof. The universal properties of $\mathbf{2}$ and $\mathbb{N}$ can be constructed in the same way as in the category Set, because the unique maps $g$ and $h$ in the diagrams below are continuous by the discreteness of $\mathbb{N}$ and $\mathbf{2}$ :
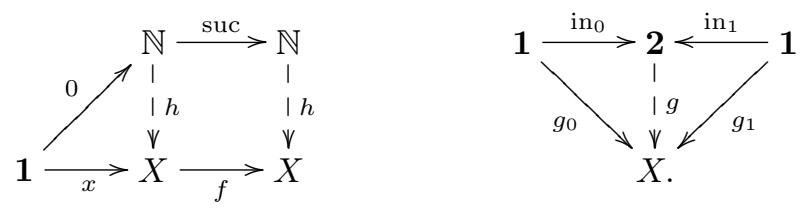

\section{The Fan Functional}

The monoid C can be regarded as a one-object category $\mathbf{C}$ with the object $\mathbf{2}^{\mathbb{N}}$ and the morphisms all uniformly continuous maps $2^{\mathbb{N}} \rightarrow 2^{\mathbb{N}}$. The Yoneda embedding $y: \mathbf{C} \rightarrow$ C-Space satisfies

$$
y\left(\mathbf{2}^{\mathbb{N}}\right)=\left(\mathbf{2}^{\mathbb{N}}, \mathrm{C}\right),
$$

where $\left(\mathbf{2}^{\mathbb{N}}, \mathrm{C}\right)$ is the $\mathrm{C}$-space corresponding to the concrete sheaf $(\mathrm{C}, \mathrm{o})$ given as an example in the previous section.

In a cartesian closed category with a natural numbers object $\mathbb{N}$ and a finite coproduct $\mathbf{2}=\mathbf{1}+\mathbf{1}$, call their exponential $\mathbf{2}^{\mathbb{N}}$ the Cantor space. With this terminology, we have that $y\left(\mathbf{2}^{\mathbb{N}}\right)$ is precisely the Cantor space in C-Space, i.e.

$$
y\left(\mathbf{2}^{\mathbb{N}}\right)=\mathbf{2}^{\mathbb{N}},
$$

where in the left-hand side $2^{\mathbb{N}}$ is the only object of the monoid $\mathrm{C}$ and in the right-hand side $\mathbf{2}^{\mathbb{N}}$ is the exponential of the two discrete spaces $\mathbb{N}$ and 2. Since 
all maps $\mathbb{N} \rightarrow \mathbf{2}$ are continuous by the discreteness of $\mathbb{N}$, the underlying set of the exponential $\mathbf{2}^{\mathbb{N}}$ is precisely the Cantor space $\mathbf{2}^{\mathbb{N}}$ (space of all maps $\mathbb{N} \rightarrow \mathbf{2}$ ). Of course one has to verify that

$$
r \in \mathrm{C} \Longleftrightarrow \forall t \in C . \forall p \in \operatorname{Probe}(\mathbb{N}) . \lambda \alpha \cdot r(t \alpha)(p \alpha) \in \operatorname{Probe}(\mathbf{2}),
$$

i.e. the two C-topologies are the same, which is routine.

As the category $\mathbf{C}$ has only one object $\mathbf{2}^{\mathbb{N}}$, the Yoneda Lemma amounts to the following.

Lemma 6 (Yoneda). For any C-space $X$, a map $\mathbf{2}^{\mathbb{N}} \rightarrow X$ is a probe on $X$ iff it is continuous.

By the Yoneda Lemma, we get that the continuous maps from the Cantor space in C-Space to the natural numbers object are in natural bijection with the uniformly continuous maps $2^{\mathbb{N}} \rightarrow \mathbb{N}$ of the meta-language used to define the model:

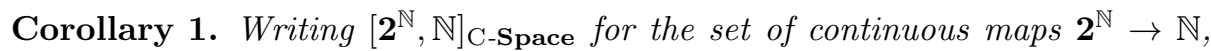
and $\operatorname{cts}\left(\mathbf{2}^{\mathbb{N}}, \mathbb{N}\right)$ for the set of uniformly continuous maps $\mathbf{2}^{\mathbb{N}} \rightarrow \mathbb{N}$, we have

$$
\left[\mathbf{2}^{\mathbb{N}}, \mathbb{N}\right]_{\text {C-Space }} \cong \operatorname{cts}\left(\mathbf{2}^{\mathbb{N}}, \mathbb{N}\right) \text {. }
$$

Moreover, the topology on $\left[\mathbf{2}^{\mathbb{N}}, \mathbb{N}\right]_{\mathrm{C}-\text { Space }}$ is discrete:

Lemma 7. The exponential $\mathbb{N}^{2^{\mathbb{N}}}$ is a discrete C-space.

Proof. Given a probe $p: \mathbf{2}^{\mathbb{N}} \rightarrow \mathbb{N}^{2^{\mathbb{N}}}$, we want to show that it is locally constant. By the construction of exponentials in Section 2, we know that for all $t, r \in \mathrm{C}$,

$$
\lambda \alpha . p(t \alpha)(r \alpha) \in \operatorname{Probe}(\mathbb{N}),
$$

i.e. $\lambda \alpha . p(t \alpha)(r \alpha)$ it is uniformly continuous. In particular, we can take

$$
t(\alpha)(i)=\alpha_{2 i} \quad \text { and } \quad r(\alpha)(i)=\alpha_{2 i+1},
$$

which are both uniformly continuous, and define $q(\alpha)=p(t \alpha)(r \alpha)$. From the proof of uniform continuity of $q$, we get its modulus $n$. (NB. Here we are implicitly using choice, but this is not a problem in intensional type theory. In a setting without choice, we would need to define uniform continuity by explicitly requiring a modulus.) Now define a map join: $\mathbf{2}^{\mathbb{N}} \times \mathbf{2}^{\mathbb{N}} \rightarrow \mathbf{2}^{\mathbb{N}}$ by

$$
\begin{array}{ll}
\operatorname{join}(\alpha, \beta)(2 i) & =\alpha_{i} \\
\operatorname{join}(\alpha, \beta)(2 i+1) & =\beta_{i} .
\end{array}
$$

Given $\alpha, \alpha^{\prime}, \beta \in \mathbf{2}^{\mathbb{N}}$ with $\alpha={ }_{n} \alpha^{\prime}$, we have

$$
\begin{aligned}
p(\alpha)(\beta) & =p(t(\operatorname{join}(\alpha, \beta)))(r(\text { join }(\alpha, \beta))) & & \text { (by the definitions of } t, r, \text { join }) \\
& =q(\text { join }(\alpha, \beta)) & & \text { (by the definition of } q) \\
& =q\left(\text { join }\left(\alpha^{\prime}, \beta\right)\right) & & \left(\text { join }(\alpha, \beta)=2 n \text { join }\left(\alpha^{\prime}, \beta\right), 2 n \geq n\right) \\
& =p\left(\alpha^{\prime}\right)(\beta) . & &
\end{aligned}
$$

Hence $p$ is locally constant and therefore $\mathbb{N}^{2^{\mathbb{N}}}$ is discrete. 
Theorem 4. There is a Fan functional

$$
\text { fan }: \mathbb{N}^{2^{\mathbb{N}}} \rightarrow \mathbb{N}
$$

in C-Space that continuously calculates moduli of uniform continuity.

Proof. Given a continuous map $f: \mathbf{2}^{\mathbb{N}} \rightarrow \mathbb{N}$, i.e. an element of $\mathbb{N}^{2^{\mathbb{N}}}$, we know $f$ is uniformly continuous as $f=f \circ \operatorname{id}_{2^{\mathbb{N}}} \in \operatorname{Probe}(\mathbb{N})$ by the continuity of $f$. Then we can get a modulus $n$ from the proof of its uniform continuity. From this $n$ we can compute the smallest modulus of $f$ as follows. We define a function $\operatorname{lmod}:\left(2^{\mathbb{N}} \rightarrow \mathbb{N}\right) \rightarrow \mathbb{N} \rightarrow \mathbb{N}$ by induction on its second argument:

$\operatorname{lmod} f 0 \quad=0$

$\operatorname{lmod} f(n+1)=$ if $\left(\forall s \in \mathbf{2}^{n} \cdot f\left(s 0^{\omega}\right) \equiv f\left(s 1^{\omega}\right)\right)$ then $(\operatorname{lmod} f n)$ else $(n+1)$.

With a proof by induction, we can show that $\operatorname{lmod} f n$ is the smallest modulus if $n$ is a modulus of $f$. Hence, we define

$$
\operatorname{fan}(f)=\operatorname{lmod} f n .
$$

According to the previous lemma, the space $\mathbb{N}^{2^{\mathbb{N}}}$ is discrete and hence this functional is continuous.

\section{Uniform Continuity of T-definable Functions}

Now we recover a well known result, using a logical relation between the settheoretical and the C-Space models of Gödel's system T.

Recall that system $\mathrm{T}$ is a simply typed lambda calculus with a ground type $\mathrm{N}$ for natural numbers and a primitive recursor rec: $\sigma \rightarrow \sigma \rightarrow \mathrm{N} \rightarrow \sigma$ for every type $\sigma$. For our purpose of formulating the uniform continuity principle, we add the binary type 2 as another ground type and a case function if: $\sigma \rightarrow \sigma \rightarrow 2 \rightarrow \sigma$ for every T type $\sigma$. Such a system can be interpreted in a cartesian closed category with a natural numbers object $\mathbb{N}$ and a coproduct $\mathbf{2}$ (or $\mathbf{1}+\mathbf{1}$ ) of two copies of the terminal object. Specifically, types are interpreted as objects: $\mathrm{N}$ is interpreted as $\mathbb{N}$, the type 2 as $\mathbf{2}$, product types as products, and function types as exponentials. Contexts are interpreted inductively as products. And a term in context is interpreted as a morphism from the interpretation of its context to the one of its type. Finally, rec and if are interpreted using the universal properties of $\mathbb{N}$ and $\mathbf{2}$.

Both the categories Set and C-Space are cartesian closed and have a natural numbers objects and a coproduct $\mathbf{1}+\mathbf{1}$; thus, they give models of system $\mathrm{T}$. Throughout this paper, we use the semantic braces $\llbracket-\rrbracket$ for the interpretation, and add Set and C-Space as subscripts to distinguish which model we are working with. Now we apply the logical relations technique to understand the relationship between these two models. 
Definition 2. The logical relation $\mathrm{R}$ over the set-theoretical and $\mathrm{C}$-Space models is defined by

1. If $\sigma$ is a $\mathrm{T}$ type, then $\mathrm{R}_{\sigma} \subset \llbracket \sigma \rrbracket_{\text {Set }} \times \llbracket \sigma \rrbracket_{\mathrm{C}-\text { Space }}$ is defined by induction on type $\sigma$ as follows:

(a) $\mathrm{R}_{\iota}\left(a, a^{\prime}\right)$ iff $a=a^{\prime}$, where $\iota$ is the ground type 2 or $\mathrm{N}$;

(b) $\mathrm{R}_{\sigma \rightarrow \tau}\left(f, f^{\prime}\right)$ iff, for any $a \in \llbracket \sigma \rrbracket_{\text {Set }}$ and any $a^{\prime} \in \llbracket \sigma \rrbracket_{\mathrm{C}-\mathrm{Space}}$, if $\mathrm{R}_{\sigma}\left(a, a^{\prime}\right)$ then $\mathrm{R}_{\tau}\left(f(a), f^{\prime}\left(a^{\prime}\right)\right)$.

2. If $\Gamma \equiv x_{1}: \sigma_{1}, \ldots, x_{n}: \sigma_{n}$ is a context, then $\mathrm{R}_{\Gamma} \subset \llbracket \Gamma \rrbracket_{\text {Set }} \times \llbracket \Gamma \rrbracket_{\text {C-Space }}$ is defined by $\mathrm{R}_{\Gamma}\left(\boldsymbol{a}, \boldsymbol{a}^{\prime}\right)$ iff $\mathrm{R}_{\sigma_{i}}\left(a_{i}, a_{i}^{\prime}\right)$ for all $i \leq n$.

3. Given $f \equiv \llbracket \Gamma \vdash t: \tau \rrbracket_{\text {Set }}$ and $f^{\prime} \equiv \llbracket \Gamma \vdash t: \tau \rrbracket_{\text {C-Space, }}, \mathrm{R}\left(f, f^{\prime}\right)$ iff, for any $\boldsymbol{a} \in \llbracket \Gamma \rrbracket_{\text {Set }}$ and any $\boldsymbol{a}^{\prime} \in \llbracket \Gamma \rrbracket_{\mathrm{C}-\text { Space }}$, if $\mathrm{R}_{\Gamma}\left(\boldsymbol{a}, \boldsymbol{a}^{\prime}\right)$ then $\mathrm{R}_{\tau}\left(f(\boldsymbol{a}), f^{\prime}\left(\boldsymbol{a}^{\prime}\right)\right)$.

With a proof by induction on types as usual, we can easily show that the interpretations of any $\mathrm{T}$ term in these two models are related.

Lemma 8. If $\Gamma \vdash t: \tau$, then $\mathrm{R}\left(\llbracket \Gamma \vdash t: \tau \rrbracket_{\text {Set }}, \llbracket \Gamma \vdash t: \tau \rrbracket_{\text {C-Space }}\right)$.

We say that an element $x \in \llbracket \sigma \rrbracket_{\text {Set }}$ in the set-theoretical model is T-definable if it is the interpretation of some closed $\mathrm{T}$ term, i.e. there exists a closed term $t: \sigma$ such that $x=\llbracket t \rrbracket_{\text {Set }}$.

Theorem 5. Any T-definable function $\mathbf{2}^{\mathbb{N}} \rightarrow \mathbb{N}$ is uniformly continuous.

Proof. If $f: \mathbf{2}^{\mathbb{N}} \rightarrow \mathbb{N}$ interprets the term $\boldsymbol{f}:(\mathrm{N} \rightarrow 2) \rightarrow \mathrm{N}$, then $f$ is related to the (uniformly) continuous map $\llbracket \boldsymbol{f} \rrbracket_{\text {C-Space }}: \mathbf{2}^{\mathbb{N}} \rightarrow \mathbb{N}$ according to the above lemma. By the definition of the logical relation, $f$ is uniformly continuous.

\section{A Continuous Realizability Semantics of HA ${ }^{\omega}$}

Recall that $\mathrm{HA}^{\omega}$ has equations between system $\mathrm{T}$ terms of the same type as atomic propositions, quantifiers that range over elements of (the interpretation of) system $\mathrm{T}$ types, and logical connectives $\wedge, \Rightarrow$ (the connectives $\vee$ and $\neg$ are definable from the other connectives). For technical convenience, we add a singleton type 1 and binary product types to the inductive definition of system $\mathrm{T}$ types. Throughout this section, we use $\sigma, \tau$ to range over $\mathrm{T}$ types, bold lower case letters $\boldsymbol{f}, \boldsymbol{x}, \boldsymbol{n}, \boldsymbol{t}, \boldsymbol{u}$ to range over $\mathrm{T}$ terms, and $\varphi, \psi$ to range over $\mathrm{HA}^{\omega}$ formulas.

With the above definition, the uniform continuity principle can be formulated in $\mathrm{HA}^{\omega}$ by the following

$$
\forall \boldsymbol{f}:(\mathrm{N} \rightarrow 2) \rightarrow \mathrm{N} . \exists \boldsymbol{n}: \mathrm{N} . \forall \boldsymbol{\alpha}, \boldsymbol{\beta}: \mathrm{N} \rightarrow 2 . \boldsymbol{\alpha}={ }_{\boldsymbol{n}} \boldsymbol{\beta} \Rightarrow \boldsymbol{f}(\boldsymbol{\alpha})=\boldsymbol{f}(\boldsymbol{\beta})
$$

where $\boldsymbol{\alpha}={ }_{\boldsymbol{n}} \boldsymbol{\beta}$ is short for $\forall \boldsymbol{i}$ :N. $\boldsymbol{i}<\boldsymbol{n} \Rightarrow \boldsymbol{\alpha}(\boldsymbol{i})=\boldsymbol{\beta}(\boldsymbol{i})$. Here we can define the relation $\boldsymbol{i}<\boldsymbol{n}$ by $\exists \boldsymbol{m}$ : N. suc $(\boldsymbol{i}+\boldsymbol{m})=\boldsymbol{n}$ where addition + is inductively defined in $\mathrm{T}$.

To any $\mathrm{HA}^{\omega}$ formula $\varphi$ we associate a type $|\varphi|$ of potential realizers. Then a continuous realizer of a formula $\Gamma \vdash \varphi$ is a pair

$$
(e, \boldsymbol{q}) \in \llbracket|\varphi| \rrbracket_{\mathrm{C} \text {-Space }} \times \llbracket \Gamma \rrbracket_{\mathrm{C} \text {-Space }} .
$$

We call this a continuous realizability semantics. In the following, semantic brackets without explicit decorations refer to the C-space interpretation. 
Definition 3 (Continuous realizability). The types of potential realizers of $\mathrm{HA}^{\omega}$ formulas are given inductively as follows:
1. $|\boldsymbol{t}=\boldsymbol{u}|=1$,
2. $|\varphi \wedge \psi|=|\varphi| \times|\psi|$,
3. $|\varphi \Rightarrow \psi|=|\varphi| \rightarrow|\psi|$,
4. $|\forall x: \sigma \cdot \varphi|=\sigma \rightarrow|\varphi|$,
5. $|\exists x: \sigma \cdot \varphi|=\sigma \times|\varphi|$.

Let $\Gamma$ be a context and $\boldsymbol{q} \in \llbracket \Gamma \rrbracket$. The relation

$(e, \boldsymbol{q})$ realizes $\Gamma \vdash \varphi$

is defined by induction on formulas as follows:

1. $(\star, \boldsymbol{q})$ realizes $\Gamma \vdash \boldsymbol{t}=\boldsymbol{u}$ iff $\llbracket \Gamma \vdash \boldsymbol{t}: \sigma \rrbracket(\boldsymbol{q}) \equiv \llbracket \Gamma \vdash \boldsymbol{u}: \sigma \rrbracket(\boldsymbol{q})$, where $\sigma$ is the type of the terms $\boldsymbol{t}$ and $\boldsymbol{u}$,

2. $(e, \boldsymbol{q})$ realizes $\Gamma \vdash \varphi_{0} \wedge \varphi_{1}$ iff $\left(\pi_{i}(e), \boldsymbol{q}\right)$ realizes $\Gamma \vdash \varphi_{i}$ for all $i \in\{0,1\}$, where $e \in \llbracket\left|\varphi_{0}\right| \rrbracket \times \llbracket\left|\varphi_{1}\right| \rrbracket$,

3. $(e, \boldsymbol{q})$ realizes $\Gamma \vdash \varphi \Rightarrow \psi$ iff for all $a \in \llbracket|\varphi| \rrbracket$ with $(a, \boldsymbol{q})$ realizing $\Gamma \vdash \varphi$, the pair $(e(a), \boldsymbol{q})$ realizes $\Gamma \vdash \psi$, where $e \in \llbracket|\psi| \rrbracket^{\llbracket|\varphi| \rrbracket}$,

4. $(e, \boldsymbol{q})$ realizes $\Gamma \vdash \forall x: \sigma . \varphi$ iff for all $a \in \llbracket \sigma \rrbracket$, the pair $(e(a),(\boldsymbol{q}, a))$ realizes $\Gamma, x: \sigma \vdash \varphi$, where $e \in \llbracket|\varphi| \rrbracket^{\llbracket \sigma \rrbracket}$ and $(\boldsymbol{q}, a) \in \llbracket \Gamma, x: \sigma \rrbracket$,

5. $(e, \boldsymbol{q})$ realizes $\Gamma \vdash \exists x: \sigma . \varphi$ iff $\left(\pi_{1}(e),\left(\boldsymbol{q}, \pi_{0}(e)\right)\right)$ realizes $\Gamma, x: \sigma \vdash \varphi$, where $e \in \llbracket \sigma \rrbracket \times \llbracket|\varphi| \rrbracket$.

We say a closed $\mathrm{HA}^{\omega}$ formula $\varphi$ is realizable if there exists $e \in \llbracket|\varphi| \rrbracket$ such that $(e, \star)$ realizes $\vdash \varphi$.

The main result of this paper is that our model validates the uniform continuity principle in the following sense.

Theorem 6. The uniform continuity principle (UC) can be realized by the Fan functional.

Proof. If $(e, \star)$ realizes UC, then $e$ is a continuous map

$$
\mathbb{N}^{2^{\mathbb{N}}} \rightarrow \mathbb{N} \times\left(\mathbf{2}^{\mathbb{N}} \rightarrow \mathbf{2}^{\mathbb{N}} \rightarrow(\mathbb{N} \rightarrow(\mathbb{N} \times 1) \rightarrow \mathbf{1}) \rightarrow \mathbf{1}\right) .
$$

By Definition 3, given any continuous $f: \mathbf{2}^{\mathbb{N}} \rightarrow \mathbb{N}$, the pair $(e(f),(\star, f))$ realizes

$$
f:(\mathrm{N} \rightarrow 2) \rightarrow \mathrm{N} \vdash \exists \boldsymbol{n}: \mathrm{N} . \forall \boldsymbol{\alpha}, \boldsymbol{\beta}: \mathrm{N} \rightarrow 2 .\left(\alpha={ }_{n} \boldsymbol{\beta} \Rightarrow \boldsymbol{f}(\boldsymbol{\alpha})=\boldsymbol{f}(\boldsymbol{\beta})\right) .
$$

We define the first component of $e(f)$ to be $\operatorname{fan}(f)$, i.e. the modulus of uniform continuity of $f$. Given $n=f a n(f)$, we want that $\left(\pi_{1}(e(f)),(\star, f, n)\right)$ realizes

$$
\boldsymbol{f}:(\mathrm{N} \rightarrow 2) \rightarrow \mathrm{N}, \boldsymbol{n}: \mathrm{N} \vdash \forall \boldsymbol{\alpha}, \boldsymbol{\beta}: \mathrm{N} \rightarrow 2 .\left(\alpha={ }_{n} \boldsymbol{\beta} \Rightarrow \boldsymbol{f}(\boldsymbol{\alpha})=\boldsymbol{f}(\boldsymbol{\beta})\right) .
$$

Given $\alpha, \beta \in \mathbf{2}^{\mathbb{N}}$ with $\alpha={ }_{n} \beta$, it is easy to verify that there exists a continuous map $e^{\prime}: \mathbb{N} \rightarrow(\mathbb{N} \times \mathbf{1}) \rightarrow \mathbf{1}$ such that $\left(e^{\prime},(\star, f, n, \alpha, \beta)\right)$ realizes

$$
\boldsymbol{f}:(\mathrm{N} \rightarrow 2) \rightarrow \mathrm{N}, \boldsymbol{n}: \mathrm{N}, \boldsymbol{\alpha}: \mathrm{N} \rightarrow 2, \boldsymbol{\beta}: \mathrm{N} \rightarrow 2 \vdash \boldsymbol{\alpha}={ }_{\boldsymbol{n}} \boldsymbol{\beta} .
$$


According to the definition of $\llbracket-\rrbracket$, we have

$$
\llbracket \Gamma \vdash \boldsymbol{f}(\boldsymbol{\alpha}): \mathrm{N} \rrbracket(\star, f, n, \alpha, \beta)=f(\alpha)
$$

and

$$
\llbracket \Gamma \vdash \boldsymbol{f}(\boldsymbol{\beta}): \mathrm{N} \rrbracket(\star, f, n, \alpha, \beta)=f(\beta)
$$

where $\Gamma \equiv \boldsymbol{f}:(\mathrm{N} \rightarrow 2) \rightarrow \mathrm{N}, \boldsymbol{n}: \mathrm{N}, \boldsymbol{\alpha}: \mathrm{N} \rightarrow 2, \boldsymbol{\beta}: \mathrm{N} \rightarrow 2$. As $n$ is the modulus of $f$, we have $f(\alpha)=f(\beta)$ and hence

$$
\llbracket \Gamma \vdash \boldsymbol{f}(\boldsymbol{\alpha}): \mathrm{N} \rrbracket(\star, f, n, \alpha, \beta)=\llbracket \Gamma \vdash \boldsymbol{f}(\boldsymbol{\beta}): \mathrm{N} \rrbracket(\star, f, n, \alpha, \beta) .
$$

Thus, $(\star,(\star, f, n, \alpha, \beta))$ realizes $\Gamma \vdash \boldsymbol{f}(\boldsymbol{\alpha})=\boldsymbol{f}(\boldsymbol{\beta})$.

\section{Construction of the Model in Intensional Type Theory}

The above results have been deliberately developed in such a way to be routinely formalizable in intensional type theory. However, certain details require a closer look. We work with an intensional type theory with a universe, $\sum$-types, $\Pi$ types, identity types and standard base types such as natural numbers, booleans, unit type and empty type.

We considered three approaches, which we developed in Agda notation [6], and are available at [24]. In the first approach, which is probably the simplest and most readable, we assumed the axiom of function extensionality,

$$
\prod_{X, Y: \text { Type }} \prod_{f, g: X \rightarrow Y}\left(\prod_{x: X} f x \equiv g x\right) \rightarrow f \equiv g,
$$

where $\equiv$ is the identity type. This approach has two drawbacks. One of them is that, because this axiom does not come with a computational interpretation, it is in principle useless for extracting computational content. In ealier stages of this work, we conjectured that the axiom of extensionality occurs only in computationally irrelevant contexts.

In order to attempt to verify that this is indeed the case, in our second approach, we made use of Agda's irrelevant fields [7], and postulated extensionality within such an irrelevant context. With this second approach, the Agda type checker proved our conjecture false. However, by refining the notion of C-topology, we were able to make it true, and our constructions and proofs type-checked. We needed to add the following condition:

(4) Any map extensionally equivalent to a probe is also a probe.

And we also needed to add more steps in each case the construction of a space was performed.

However, a second drawback remains in the two approaches considered above: they do not seem to allow a construction of the Fan functional. To define a continuous Fan functional in the model (Section 3), we derive its continuity by 
showing that its domain, the space $\mathbb{N}^{2^{\mathbb{N}}}$, is discrete, i.e. by showing that any probe $p: \mathbf{2}^{\mathbb{N}} \rightarrow \mathbb{N}^{2^{\mathbb{N}}}$ is locally constant. We can find an $n \in \mathbb{N}$ and show that for any $\alpha, \alpha^{\prime} \in \mathbf{2}^{\mathbb{N}}$ with $\alpha={ }_{n} \alpha^{\prime}$, the two maps $p(\alpha), p\left(\alpha^{\prime}\right): \mathbf{2}^{\mathbb{N}} \rightarrow \mathbb{N}$ are equal using functional extensionality. However, this does not allow us to conclude that their proofs of continuity are also equal, in order to conclude $p(\alpha) \equiv p\left(\alpha^{\prime}\right)$, and the proofs of continuity cannot be put in an irrelevant field because they are computationally relevant, at least not without further thought.

In our third and last, fully successful, approach, instead of assuming any form of extensionality or irrelevant fields, we slightly adjusted the definition of C-space. Now a C-topology is defined on a set equipped with an equivalence relation, that is, on a setoid. With this, we can define a notion of equality of continuous functions that ignores continuity proofs, and the Fan functional can be implemented as discussed in the previous sections. We remark that the probe axiom (4) mentioned above is still needed.

This third approach works well, and does not need to assume any nonstandard axiom for intensional type theory. However, the drawback is that the proofs are much less readable than in the first two approaches. It would be desirable to find an approach that avoids setoids and addresses the equality problem for continuous functions by hiding information in the definition of continuity without losing computational information to obtain a more concise formalization.

At the moment we formalized everything discussed above, including the Fan functional, the set and continuous interpretations of system $\mathrm{T}$, their logical relation, and the proof that the set-theoretical definable functions $2^{\mathbb{N}} \rightarrow \mathbb{N}$ are uniformly continuous, but excluding the definition of $\mathrm{HA}^{\omega}$ and its interpretation, which is under development.

\section{Future Work}

Both the category of sheaves and its full subcategory of C-spaces are locally cartesian closed. For the second category, an exponential in a slice category C-Space $/ X$ is constructed in the same way as the one in the slice category Set $/ X$, with a suitable construction of the topology on its domain (see [2, Proposition 43]). Thus, rather than giving a realizability interpretation of UC, we can understand its quantifiers as $\prod$ and $\sum$, and interpret them using the locally cartesian closed structure $[21,10,15,11]$. With a cursory calculation to be fully verified in future work, we can show that the Fan functional (modulo some type isomorphisms) is an element of the interpretation of UC. Hence our development seems to generalize from system $\mathrm{T}$ to dependent types. We have not considered the interpretation of universes with our continuous model so far.

As mentioned in the introduction, we conjecture that the system $\mathrm{T}$ model consisting of C-spaces is classically equivalent to the model of Kleene-Kreisel functionals, and hence can be seen as a constructive development of that model. 


\section{References}

1. S. Awodey and A. Bauer. Propositions as [types]. Journal of Logic and Computation, 14(4):447-471, 2004.

2. J. C. Baez and A. E. Hoffnung. Convenient categories of smooth spaces, 2008.

3. A. Bauer, L. Birkedal, and D. Scott. Equilogical spaces. Theoret. Comput. Sci., 315(1):35-59, 2004.

4. A. Bauer and A. Simpson. Continuity begets continuity. Presented at Trends in constructive mathematics in Germany, 2006.

5. M. J. Beeson. Foundations of Constructive Mathematics. Springer-Verlag, 1985.

6. A. Bove and P. Dybjer. Dependent types at work. Proceedings of Language Engineering and Rigorous Software Development, LNCS, 5520:57-99, 2009.

7. Community. Agda wiki. http://wiki.portal.chalmers.se/agda/.

8. T. Coquand and G. Jaber. A note on forcing and type theory. Fundamenta Informaticae, 100(1-4):43-52, 2010.

9. T. Coquand and G. Jaber. A computational interpretation of forcing in type theory. In Epistemology versus Ontology, volume 27, pages 203-213. Springer Netherlands, 2012.

10. P.-L. Curien. Substitution up to isomorphism. Fundamenta Informaticae, 19(1/2):51-85, 1993.

11. P. Dybjer. Internal type theory. In Types for proofs and programs (Torino, 1995), volume 1158 of Lecture Notes in Comput. Sci., pages 120-134. Springer, 1996.

12. M. Escardó, J. Lawson, and A. Simpson. Comparing Cartesian closed categories of (core) compactly generated spaces. Topology Appl., 143(1-3):105-145, 2004.

13. M. P. Fourman. Notions of choice sequence. In The L. E. J. Brouwer Centenary Symposium Proceedings of the Conference held in Noordwijkerhout, volume 110, pages $91-105,1982$.

14. M. P. Fourman. Continuous truth I, non-constructive objects. In Proceedings of Logic Colloquium, Florence 1982, volume 112, pages 161-180. Elsevier, 1984.

15. M. Hofmann. On the interpretation of type theory in locally Cartesian closed categories. In Computer science logic (Kazimierz, 1994), volume 933 of Lecture Notes in Comput. Sci., pages 427-441. Springer, Berlin, 1995.

16. P. T. Johnstone. On a topological topos. Proceedings of the London Mathematical Society, 38(3):237-271, 1979.

17. P. T. Johnstone. Sketches of an Elephant: A Topos Theory Compendium. Oxford University Press, 2002.

18. S. Mac Lane and I. Moerdijk. Sheaves in Geometry and Logic: A First Introduction to Topos Theory. Springer, 1992.

19. D. Normann. Recursion on the countable functionals, volume 811 of Lec. Not. Math. Springer, 1980.

20. D. Normann. Computing with functionals - computability theory or computer science? Bull. Symbolic Logic, 12(1):43-59, 2006.

21. R. A. G. Seely. Locally cartesian closed categories and type theory. Mathematical Proceedings of the Cambridge Philosophical Society, 95(1):33-48, 1984.

22. E. H. Spanier. Quasi-topologies. Duke Mathematical Journal, 30(1):1-14, 1963.

23. G. van der Hoeven and I. Moerdijk. Sheaf models for choice sequences. Annals of Pure and Applied Logic, 27(1):63-107, 1984.

24. C. Xu and M. H. Escardó. A constructive model of uniform continuity, developed in Agda. Available at the authors' institutional web pages, 2012-2013. 